Canadian University Music Review

Revue de musique des universités canadiennes

\title{
Michel Chion. Guide des objets sonores. Préface de Pierre \\ Schaeffer. Paris : INA-GRM, Buchet-Chastel, 1983, 187 pp.
}

\section{Francis Dhomont}

Numéro 5, 1984

URI : https://id.erudit.org/iderudit/1014023ar

DOI : https://doi.org/10.7202/1014023ar

Aller au sommaire du numéro

Éditeur(s)

Canadian University Music Society / Société de musique des universités

canadiennes

\section{ISSN}

0710-0353 (imprimé)

2291-2436 (numérique)

Découvrir la revue

Citer ce compte rendu

Dhomont, F. (1984). Compte rendu de [Michel Chion. Guide des objets sonores. Préface de Pierre Schaeffer. Paris : INA-GRM, Buchet-Chastel, 1983, 187 pp.]

Canadian University Music Review / Revue de musique des universités

canadiennes, (5), 366-368. https://doi.org/10.7202/1014023ar

All Rights Reserved (c Canadian University Music Society / Société de musique des universités canadiennes, 1984
Ce document est protégé par la loi sur le droit d'auteur. L’utilisation des services d'Érudit (y compris la reproduction) est assujettie à sa politique d'utilisation que vous pouvez consulter en ligne.

https://apropos.erudit.org/fr/usagers/politique-dutilisation/ 
Michel Chion. Guide des objets sonores. Préface de Pierre Schaeffer. Paris : INA-GRM, Buchet-Chastel, 1983, $187 \mathrm{pp.}$

Annoncé depuis dix ans et attendu par beaucoup, ce livre, à la fois décryptage et exégèse du Traité des objets musicaux de Pierre Schaeffer, ne décevra pas : il tient ses promesses et nous semble même les dépasser en ne se limitant pas à une simple nomenclature de termes. Et à cet égard, il n'est pas sans signification que le titre initialement prévu de Lexique ait été abandonné au profit de celui de Guide qui souligne l'ambition de son auteur d'offrir "beaucoup plus que l'index des mots clés qui manquait au Traité » (Chion 1976 : 43).

Ainsi, nous sommes avertis : on se propose de nous frayer un chemin à travers le maquis luxuriant et touffu d'un ouvrage fondamental. Et en effet, le Guide des objets sonores, malgré sa fidélité au Traité de Schaeffer, n'en est pas le résumé : «il résulte d'un long et important travail de dépouillement (...) », affirme Chion que l'on croit volontiers, "(...) et de reformulation (...)" (p. 13). Ce livre devra remplir trois fonctions, nous dit encore son auteur : celle d'un Index, celle d'un Dictionnaire, celle d'un Guide de lecture "reprenant à grands traits, dans une progression raisonnée, les grands thèmes du Traité " (p. 14).

Il est donc possible de cette façon de se reporter au T.O.M.' lui-même grâce à l'index figurant à la fin de chaque article du Guide, mais également d'accéder aux thèses et notions clés du Traité, prélevées et commentées par Michel Chion, en se référant à une Table de consultation alphabétique (p. 16), ou enfin de "suivre le Guide " pas à pas. Ce dernier itinéraire comporte alors cinq étapes : la première dégage la notion d'objet sonore; la seconde pose la question d'une musique généralisée avec son corollaire, véritable épine dorsale du T.O.M. : musicalité/sonorité; la troisième trace les grandes lignes d'une recherche musicale et en définit le programme; la quatrième (Typologie) et la cinquième (Morphologie) détaillent les opérations et les critères requis pour l'identification, le classement et la description des objets sonores. Suivent en annexe cinq tableaux synoptiques (pp. 168 à 177), choisis dans le Traité par Chion, qui récapitulent les principaux concepts développés par Pierre Schaeffer.

Précisons que le travail de Michel Chion opère, comme il le souligne lui même, une coupe synchronique - véritable " arrêt sur l'image » - dans le déferlement diachronique de la pensée schaefferienne, ce qui en fige peut-être un peu la dynamique mais permet, en revanche, l'appréhension rapide des mille ramifications d'un questionnement toujours sur la brèche. Et c'est bien là, à notre avis, l'un des principaux mérites de ce livre dont l'efficacité didactique et la simplicité d'accès exigeait qu'eût été faite une lecture préalable proche de l'herméneutique (terme qui ne nous paraît pas trop fort et qui souhaite rendre hommage au maître comme au disciple), notamment des livres II et IV du T.O.M., 
les moins consultés probablement, bien que les plus révélateurs d'une réflexion novatrice.

Il ne fait aucun doute que Chion possède son Schaeffer sur le bout du doigt et que sa longue intimité avec le Traité lui permet, les concevant bien, d'énoncer clairement des notions demeurées, aujourd'hui encore, énigmatiques pour beaucoup. Il a le sens des formules simples (mais pas simplistes) qui jettent une lumière nouvelle sur des idées que l'on croyait bien connaître ou qui, au contraire, étaient restées peu accessibles. Ses gloses évitent toujours le pléonasme et le jargon, ce qui en rend la lecture fluide malgré l'entrelacs des thèmes abordés; le style, alerte, n'est jamais cuistre et se veut convaincant. Le souci d'offrir un ouvrage fonctionnel, utilitaire, un outil de travail opérationnel est évident; il apparaît dans chaque rubrique, ici dans l'insistance à dissiper l'ambiguïté d'un terme à double sens (e.g. : anamorphose (p. 24), critère (p. 121), etc.), là dans le soin apporté à rendre lisible tel tableau aux entrées multiples (p. 26) ou même à concevoir de nouveaux schémas ou énoncés mnémoniques que l'on interrogera avec profit (pp. 60, 67, $94,147, \ldots$ ), ainsi que des noms de code, sacrifice sans doute à la mode des sigles et acronymes mais qui, à l'usage, s'avèrent plus évocateurs, plus simples à mémoriser que les habituels renvois numérotés (BIFINTEC, PROGREMU, LOI PCV2, etc.).

En optant pour une certaine hiérarchisation des grandes idées du Traité - sans pour autant «ne retenir que celles qui pouvaient être jugées éprouvées et indiscutables" (p. 13) -, en mettant en évidence ses principaux linéaments, cette étude, apparemment exhaustive, plante les jalons qui repèrent notre lecture.

On pourra regretter toutefois que Chion, par respect excesssif peut-être, se soit imposé de marcher dans les traces du grand homme et n'ait pas pris la liberté, malgré l'emploi de quelques conditionnels, de soumettre cette ouvre, pour aussi imposante qu'elle soit et dans l'intérêt même du message qu'elle contient, à l'épreuve d'une analyse critique. On souhaiterait en effet que certaines notions, notamment celle, normative, d'objet convenable (p. 97-98) fassent l'objet d'un examen plus acéré : "Les critères proposés par l'auteur (Schaeffer) sont nécessairement subjectifs et ne sont pas partagés par tout le monde ", écrivait J.-J. Nattiez à juste titre en 1976 à propos du T.O.M. ${ }^{2}$. À l'occasion de l'actualisation de ce grand livre, l'expérience du compositeur Michel Chion aurait pu relancer le débat dans " une direction tournée moins vers l'observation que vers l'action" ". Peut-être le disciple, fidèle, certes, mais praticien aurait-il dû prolonger la réflexion de son maître en ébauchant les fondements de ce fameux Traité des organisations que Schaeffer semblait appeler de ses voux dans la postface à la réédition du T.O.M.

Il y a huit ans, il est vrai, Chion prenait déjà soin de préciser que son Lexique ne serait "pas encore l'étude critique " du Traité (1976:43). Cet adverbe nous permet d'espérer qu'une suite prenant appui sur la réalité du répertoire contemporain sera donnée au présent ouvrage. 
D'autre part, on peut craindre que ce Guide, malgré les objectifs qu'il se fixe et les avertissements de son auteur, n'intéresse - comme cela fut dit à tort - que ceux qu'intéresse la musique concrète et demeure, comme le Traité, "la Bible d'une secte fermée "(Chion). Ce serait alors entretenir une fois de plus le malentendu qui pèse sur le T.O.M. depuis sa sortie en 1966 et ignorer que l'une des raisons d'être de ces deux ouvrages est d'inviter le lecteur à la pratique de l'acoulogie (p. 94) qui n'est autre que l'art d'écouter.

Disons pour conclure que cette contribution à l'ouvre de Schaeffer et plus généralement à la recherche de ce que l'on pourrait nommer les universaux de la musique, constitue, malgré ses dimensions volontairement modestes, un ouvrage de référence qui devrait figurer dans la bibliothèque de tous ceux (musiciens, cinéastes, gens de théâtre, de radio, de télévision, etc.) " qui sont confrontés de toutes les manières avec l'univers des sons ${ }^{5} »$. Michel Chion regrettait qu'aucun disciple de Schaeffer ne lui eût élevé un monument filial. Eh! bien, voilà qui est fait.

Francis Dhomont

\section{NOTES}

1. C'est l'abréviation courante du Traité des ()bjets Musicaux.

2. Dans une note de J.-J. Nattiez in "Ce que le G.R.M. pense du T.O.M. ", propos recueillis par François Delalande, Cahiers Recherche/Musique, N" 2 , 1976, p. 28.

3. François Bayle, ibid., p. 33

4. Souligné par nous.

5. Selon une expression qu'on relève dans le prière d'insérer du livre.

\section{RÉFÉRENCES}

CHION, M.

1976: "Le Traité et son double ou un petit lexique pour un grand T.O.M. ", Cahiers Recherche/Musique, N" 2. 43-54. 